\title{
Robotic mitral valve repair: standardized repair strategy ensures consistent results
}

\author{
Hikaru Ishii ${ }^{1,2}$, Mao Ting ${ }^{1}$, Nai-Hsin $\mathrm{Chi}^{1}$ \\ ${ }^{1}$ Department of Surgery, National Taiwan University Hospital and National Taiwan University College of Medicine, Taipei, Taiwan; ${ }^{2}$ Department of \\ Cardiovascular Surgery, Kyorin University School of Medicine, Kyorin University, Tokyo, Japan \\ Correspondence to: Nai-Hsin Chi, MD. No.7, Chung Shan S. Rd., Zhongzheng Dist., Taipei City 10002, Taiwan. Email: chinaihsin@gmail.com.
}

Submitted Jun 22, 2018. Accepted for publication Oct 23, 2018.

doi: $10.21037 /$ acs.2018.10.11

View this article at: http://dx.doi.org/10.21037/acs.2018.10.11

\section{Clinical vignette}

Surgical repair offers long-term mitral valve competence in degenerative mitral valve disease. To achieve a better outcome, reduce pain, limit the wound size and shorten the time to recovery, we use a robotic total endoscopic approach for mitral valve repair. To ensure consistent surgical results, we standardized the repair strategy as a conventional sternotomy method without compromising the repair technique $(1,2)$.

A 63-year-old man presented for management of severe mitral regurgitation (MR). One year prior to presentation, he had suspected infective endocarditis that was medically managed with an extended course of intravenous antibiotics. Over the preceding 6 months, he reported a decreasing exercise tolerance. Physical examination revealed blood pressure of 130/73 $\mathrm{mmHg}$ and pulse of $62 \mathrm{bpm}$. A grade V/VI holosystolic murmur was detected at the fifth left intercostal space, radiating to the axilla. Transthoracic echocardiography (TTE) demonstrated a dilated left atrium measuring $48 \mathrm{~mm}$, left ventricular ejection fraction (LVEF) of $60 \%$ and right ventricular systolic pressure of $23 \mathrm{mmHg}$. Severe MR and mild tricuspid regurgitation with normal aortic and pulmonic valves were observed. The patient underwent pre-operative screening for robotic mitral valve repair, including coronary angiography to exclude coronary artery disease and computed tomography to ensure feasibility and safety of peripheral perfusion.

\section{Surgical techniques}

\section{Standardized artificial chordae for leaflet prolapse}

We routinely employ a non-resection strategy for mitral valve repair. Flail leaflets caused by ruptured chordae or leaflet prolapse can be repaired with this method. A 4-0 Gore-Tex suture neo-chord is fixed to the papillary muscle; the double-armed suture is passed twice through a healthy portion of the papillary muscle head. The neo-chord is then sited in the prolapsed leaflet at its margin by passing twice through the leaflet edge. Two passes are necessary due to the slippery property of Gore-Tex suture, but it is also useful in facilitating length adjustment. Neo-chord length is determined according to annulus height. The repaired leaflet should have the same height as the annulus, using adjacent normal leaflet height as a reference. We have some preferences for repair using a neo-chord: First, we seldom extend chords across the midline. If the patient has a valve lesion that extends across the midline, we prefer to use separate neo-chords on each side. Second, if after neochord implantation there is any large cleft or indentation, it is closed.

\section{Mitral valve repair}

The mitral valve is exposed. For this patient, an A2 and A3 segment prolapse and chord rupture over the A3 segment was determined. In addition, posteromedial commissural leaflet prolapse was noted. First, we exposed the papillary muscle and created an artificial chord on the papillary muscle head, then fixed the neo-chord on the A2 and A3 segments. The length of artificial chords was decided by the height of the annulus and compared to the adjacent normal leaflet height. The other new paired artificial chord was placed on the medial papillary muscle and fixed to the prolapsed commissural leaflet. After completing the neochord implant, the annuloplasty ring was measured. We 
used a 36-mm Physio-Ring with 12 interrupted sutures. A magic stitch was placed over the medial commissure and A3 segment. The repair was deemed complete following assessment with saline insufflation to fill and pressurize the left ventricle.

\section{Pearls and pitfalls}

The way to anchor neochords on papillary muscle and prolapsed leaflet should be standardized. The concept can be applied on every prolapse or rupture chords. The annuloplasty ring sizing, annulus suture and the way to secure ring should also be standardized. By protocoled each repair steps, the whole team can be easily familiar with every repair procedure and ensure safety and outcome of the patient.

\section{Completion}

Transesophageal echocardiography (TEE) showed no MR and adequacy of de-airing was confirmed with the patient off cardiopulmonary bypass before de-cannulation. Once the heart was beating, the antegrade cardioplegia catheter was removed from the aorta and the puncture site was closed with a pledget-reinforced 4-0 Gore-Tex suture. Intraoperative TEE demonstrated no MR. The postoperative hospital course was without complications. TTE at discharge demonstrated normal LV function (LVEF $=65 \%$ ), no MR and a normal aortic valve. The operative time was two hours and 35 minutes. Blood loss was less than $50 \mathrm{~mL}$.

\section{Comments}

The total endoscopic treatment option for mitral valve surgery can be achieved using a robotic system. The current surgical aim in mitral valve surgery should be not only to achieve long-term mitral valve competence, but also to reduce pain, limit wound size, and shorten the time to recovery $(3,4)$. To achieve this goal, traditional mitral repair methods can be adapted to robotic surgery without any compromise of the outcome. In addition, the surgical risks should be diminished as much as possible. Accordingly, we standardized every step in our robotic mitral repair method. Between January 2012 and December 2017, we performed 347 robotic mitral repair surgeries. At five postoperative years, the survival rate was $98.2 \%$, with $98.5 \%$ freedom from reoperation and $98 \%$ freedom from more than grade II MR. We used a standardized valve repair strategy as for a traditional open method to ensure consistent long-term results. By standardizing every repair step, the results were consistent and the outcomes were good.

\section{Acknowledgements}

The authors thank the staff at the Seventh Core Laboratory, Department of Medical Research, National Taiwan University Hospital for their technical support. We would especially like to thank Yi-Jia Liou for preparing the figures.

\section{Footnote}

Conflict of Interest: The authors have no conflicts of interest to declare.

\section{References}

1. Chi NH, Huang CH, Huang SC, et al. Robotic mitral valve repair in infective endocarditis. J Thorac Dis 2014;6:56-60.

2. Gillinov AM, Mihaljevic T, Javadikasgari H, et al. Early results of robotically assisted mitral valve surgery: Analysis of the first 1000 cases. J Thorac Cardiovasc Surg 2018;155:82-91.e2.

3. Cao C, Harris C, Croce B, et al. Robotic mitral valve surgery. Ann Cardiothorac Surg 2017;6:73.

4. Cao C, Wolfenden H, Liou K, et al. A meta-analysis of robotic vs. conventional mitral valve surgery. Ann Cardiothorac Surg 2015;4:305-14.
Cite this article as: Ishii H, Ting M, Chi NH. Robotic mitral valve repair: standardized repair strategy ensures consistent results. Ann Cardiothorac Surg 2018;7(6):837-838. doi: 10.21037/acs.2018.10.11 